\title{
GEOMETRIC AUTOMORPHISMS OF A FREE GROUP OF RANK AT LEAST THREE ARE RARE
}

\author{
S. M. GERSTEN
}

\begin{abstract}
The characteristic polynomials of geometric automorphisms of a free
\end{abstract} group of finite rank at least three form a nowhere dense set in the Zariski topology.

1. Introduction. Call an automorphism $\phi$ of a free group $F$ of finite rank geometric if there exists a two dimensional manifold $M$, compact and bounded (but not necessarily orientable) and a homeomorphism $h$ of the pair $(M, x), x \in M$, such that the map on $\pi_{1}(M, x)$ induced by $h$ is $\phi$. Stallings [St] gave the first example of a nongeometric automorphism.

The map $\phi$ above induces an automorphism $\phi_{\mathrm{ab}}$ of $F_{\mathrm{ab}}$, the abelianization of $F$. Let $\operatorname{char} \phi=\operatorname{det}\left(x \cdot 1-\phi_{\mathrm{ab}}\right) \in \mathbf{Z}[x]$, the characteristic polynomial of $\phi_{\mathrm{ab}}$. It is monic and has constant term \pm 1 . Stallings' result (which is proved but not stated explicitly in $[\mathbf{S t}])$ is

THEOREM. If for any pair $(\lambda, \mu)$ of roots of char $\phi$ and integer $r>0$ one has $(\lambda \mu)^{r} \neq 1$, then $\phi$ is not geometric.

We shall show that Stallings' condition $(\lambda \mu)^{r} \neq 1$ is generic if rank $F \geqslant 3$, in the sense that it defines an open dense subset of the polynomials $p(x) \in \mathbf{Z}[x]$ which are monic and have $p(0)= \pm 1$. The geometric automorphisms are rare (Bourbaki's terminology) in the sense that their characteristic polynomials form a nowhere dense subset (Anglo-Saxon terminology [D, p. 621]), when rank $F \geqslant 3$.

The case rank $F=2$ must be excluded since it is known that any automorphism $\phi$ of a free group of rank 2 is geometric (compare e.g. [L-S, p. 25, Proposition 4.5]). Note that the eigenvalues $\lambda, \mu$ of $\operatorname{char} \phi$ satisfy $(\lambda \mu)^{2}=1$, so Stallings' necessary condition to be geometric is automatically satisfied here.

The $P V$ automorphisms introduced in [St] to give explicit examples of nongeometric automorphisms enter here also although through the back door, so to speak. One uses the existence of certain $P V$ numbers to show that Stallings' condition $(\lambda \mu)^{r} \neq 1$ is nonvacuous. It can be satisfied in every degree $\geqslant 3$ by a suitable monic polynomial $p(x) \in \mathbf{Z}[x]$ with $p(0)=-1$.

Received by the editors December 22, 1982.

1980 Mathematics Subject Classification. Primary 20F28, 20E36, 57N05, 12A15.

$K e y$ words and phrases. Free group, automorphism, geometrically realizable, $P V$ number, nowhere dense set.

(C) 1983 American Mathematical Society $0002-9939 / 82 / 0000-1449 / \$ 02.50$ 
2. Stallings' condition. Let $\mathscr{P}_{n}$ denote the set of polynomials $p(x) \in \mathbf{Z}[x]$ which are monic and have $p(0)= \pm 1$. Denote by $\mathscr{P}_{n}(Q)$ the polynomials in $Q[x]$ which are monic and have constant term \pm 1 . We observe that $\mathscr{P}_{n}(Q) \cong Q^{n-1} \times\{ \pm 1\} \subset Q^{n}$, affine space over $Q$ of dimension $n$, where the isomorphism is given by

$$
p(x)=x^{n}+a_{1} x^{n-1}+\cdots+a_{n} \mapsto\left(a_{1}, a_{2}, \ldots, a_{n}\right) \quad\left(a_{n}= \pm 1\right) .
$$

Topologize $\mathscr{P}_{n}(Q)$ by means of this bijection as a subspace of the Zariski topology on $Q^{n}$. Recall that a basis for the open sets of this topology is the collection $D(f)$, where $f \in Q\left[x_{1}, \ldots, x_{n}\right]$, and $D(f)=\left\{\left(a_{1}, \ldots, a_{n}\right) \in Q^{n} \mid f\left(a_{1}, \ldots, a_{n}\right) \neq 0\right\}$.

Observe that each of the two connected components $\stackrel{P}{n}_{n}^{\varepsilon}(Q) \cong Q^{n-1} \times\{\varepsilon\}, \varepsilon= \pm 1$, is isomorphic with $Q^{n-1}$. Let $\mathscr{P}_{n}^{\varepsilon}=\mathscr{P}_{n}^{\varepsilon}(Q) \cap \mathscr{P}_{n}$.

Suppose now that $p \in \mathscr{P}_{n}(Q), p=x^{n}+a_{1} x^{n-1}+\cdots+a_{n}$. We may factor $p$ in $\bar{Q}[x]$ as $p=\prod_{i=1}^{n}\left(x-\lambda_{i}\right)$. For each integer $r \geqslant 1$ define

$$
p_{r}(x)=: \prod_{i=1}^{n}\left(x-\lambda_{i}^{r}\right)=x^{n}+a_{1}(r) x^{n-1}+\cdots+a_{n}(r) \text {. }
$$

LEMMA 2.1. $p_{r}(x) \in Q[x]$ and $a_{n}(r)= \pm 1$. In addition if $p(x) \in P_{n}$, then $p_{r}(x) \in$ $\mathscr{P}_{n}$.

Proof. The $a_{j}(r)$ are elementary symmetric functions of the $\lambda_{1}^{r}, \ldots, \lambda_{n}^{r}$, so lie in $Q$. In addition they are algebraic integers if $a_{i} \in \mathbf{Z}$, whence $a_{i}(r) \in \mathbf{Z}$ in this case. Finally $a_{n}(r)=(-1)^{n}\left(\lambda_{1} \cdots \lambda_{n}\right)^{r}=(-1)^{n r+n}\left((-1)^{n} \lambda_{1} \cdots \lambda_{n}\right)^{r}=(-1)^{n r+n} a_{n}^{r}= \pm 1$.

If $p \in \mathscr{P}_{n}(Q)$, let $\tilde{p}=p(0) \cdot x^{n} \cdot p(1 / x)$. It is clear that $\tilde{p} \in \mathfrak{P}_{n}(Q)$. In addition the operation $p \mapsto \tilde{p}$ preserves $\mathscr{P}_{n}$.

LEMMA 2.2. A necessary and sufficient condition that $p \in P_{n}(Q)$ have a pair of roots $(\lambda, \mu)$ with $\lambda \mu=1$ is that $R(p, \tilde{p})=0$, where $R(f, g)$ is the resultant of two polynomials $f$ and $g$.

Proof. See [W, §27].

COROllaRY 2.3. A polynomial $p \in \mathscr{P}_{n}(Q)$ has a pair of roots $(\lambda, \mu)$ with $(\lambda \mu)^{r}=1$ for some $r \geqslant 1$ iff for some $r \geqslant 1, R\left(p_{r}, \tilde{p}_{r}\right)=0$.

Observe that by 2.1 and the theory of symmetric functions $R\left(p_{r}, \tilde{p}_{r}\right)$ is a polynomial function of the coefficients $a_{1}, a_{2}, \ldots, a_{n}$ of $p$.

We can now state our main result.

THEOREM 2.4. There exists an $N=N(n)$ such that $p \in \mathscr{P}_{n}(Q)$ has a pair of roots $(\lambda, \mu)$ satisfying $(\lambda \mu)^{r}=1$ for some $r \geqslant 1$ if and only if $\prod_{i=1}^{N} R\left(p_{i}, \tilde{p}_{i}\right)=0$.

Proof. We recall that $\lim _{n \rightarrow \infty} \phi(n)=\infty$ where $\phi(n)$ is Euler's function, the number of units in $\mathbf{Z}_{n}$. (Here is a clever argument due to Fletcher Gross. $\phi(n) \geqslant$ $\pi(n)-\pi(n / 2)-1$ since a prime number $p$ satisfying $n / 2<p<n$ must be relatively prime to $n$. The desired limit follows from the prime number theorem.) It follows that there exists $N=N(n)$ such that $\phi(m)>n$ ! for $m>N$.

If $(\lambda \mu)^{r}=1$, then $\lambda \mu$ is an $r$ th root of unity. Since $\lambda, \mu$ lie in a splitting field of $p \in Q[x]$, the $r$ th root of unity $\lambda \mu$ must be of degree $\leqslant n$ ! over $Q$. If the order of $\lambda \mu$ 
is precisely $r_{1}$ (so $\lambda \mu$ is a primitive $r_{1}$ th root of unity) then $\phi\left(r_{1}\right) \leqslant n$ ! Thus $\left(\lambda^{r_{1}}, \mu^{r_{1}}\right)$ is an inverse pair of roots of $p_{r_{1}}$ and $r_{1} \leqslant N$. Thus $R\left(p_{r_{1}}, \tilde{p}_{r_{1}}\right)=0$ with $r_{1} \leqslant N$. This completes the proof of 2.4 .

As an example, if $n=3$, then $N=N(3)=18$, which is impractically large.

Corollary 2.5. The Stallings' condition " $(\lambda \mu)^{r}=1$ for some $r \geqslant 1$ " defines a Zariski closed subset $Z$ of $\mathscr{P}_{n}(Q)$ and hence a closed subset of $\mathscr{P}_{n}$ in the subspace topology.

Corollary 2.5 leaves open the question whether or not $Z \cap \mathcal{P}_{n}^{\varepsilon}$ is a proper closed subset of $\mathscr{P}_{n}^{\varepsilon}$. Indeed, if $n=2, Z \cap \mathscr{P}_{n}^{\varepsilon}=\mathscr{P}_{n}^{\varepsilon}$ as we observed in the introduction.

3. $P V$ numbers. A $P V$ number is an algebraic integer $\theta$ such that $|\theta|>1$ but all the other conjugates of $\theta$ lie in the interior of the unit circle. The following properties of $P V$ numbers are immediate.

3.1. If $\theta$ is $P V$, so is $\theta^{r}, r \geqslant 1$.

3.2. If $p(x)$ is the monic minimal polynomial satisfied by the $P V$ number $\theta$ then $p(x) \in \mathbf{Z}[x]$. If $\theta$ is a unit (in the algebraic integers) then $p(0)= \pm 1$.

3.3. If the degree over $Q$ of the $P V$ number $\theta$ is at least 3 , then $(\lambda \mu)^{r} \neq 1$ for any $r \geqslant 1$. Here $(\lambda, \mu)$ is any pair of roots of the minimal polynomial $p$ of $\theta$.

LEMMA 3.4. If for each $n \geqslant 3$ there is a PV number $\theta_{n}$ of degree $n$ over $Q$ such that for even $n$ the norm of $\theta_{n}$ is -1 , then $Z \cap{ }_{n}^{p}(Q)$ is a proper closed subset of $P_{n}^{\varepsilon}(Q)$, in the notation of $2.5(\varepsilon= \pm 1)$.

Proof. Let the conjugates of $\theta_{n}$ be $\theta_{n}=\alpha_{1}, \alpha_{2}, \ldots, \alpha_{n}$ and let $p(x) \in \mathbf{Z}[x]$ be the minimal polynomial of $\theta_{n}$. If $n$ is odd, then $p(x)$ and $(-1)^{n} p(-x)$ are in $\mathbf{Z}[x]$, are monic, and are such that one of them has constant term -1 and the other has constant term +1 . Since the roots of the second are $-\alpha_{1}, \ldots,-\alpha_{n}$, it follows from 3.3 that $(\lambda \mu)^{r} \neq 1, r \geqslant 1$, for any pair of roots either of $p(x)$ or of $(-1)^{n} p(-x)$. Thus ${ }_{n}^{(P)}(Q) \cap Z \neq\left({ }_{n}^{f}(Q)\right.$ for $\varepsilon= \pm 1$ if $n$ is odd.

On the other hand if $n$ is even and $\theta_{n}$ has norm -1 , then the constant term of $p(x)$ is -1 , so 3.3 shows that $Z \cap \cdot{ }_{n}{ }_{n}^{-1}(Q) \neq \cdot ?_{n}^{-1}(Q)$.

Next consider $\theta_{n}^{2}$ with conjugates $\alpha_{1}^{2}, \alpha_{2}^{2}, \ldots, \alpha_{n}^{2}$. By $3.1, \theta_{n}^{2}$ is also $P V$ and its norm is 1 . Thus the constant term of the minimal polynomial of $\theta_{n}^{2}, p_{2}(x)=\prod_{i=1}^{n}\left(x-\alpha_{i}^{2}\right)$, is 1. Again 3.3 shows that $Z \cap \mathscr{P P}_{n}^{+1}(Q) \neq \mathscr{P}_{n}^{+1}(Q)$.

To be able to apply 3.4 , we need a supply of $P V$ numbers.

TheOREM [Si]. For each $n \geqslant 1$ the polynomial

$$
P_{n}(x)=x^{n}\left(x^{2}-x-1\right)+\left(x^{2}-1\right)
$$

has a root $\theta_{n+2}$ which is a $P V$ number of degree $n+2$.

Observe that for $n$ even, the norm of $\theta_{n}$ is -1 , so the numbers $\theta_{n}$ satisfy the hypothesis of 3.4 .

Hence we deduce

THEOREM 3.5. For each $n \geqslant 3, Z \cap \mathscr{P}_{n}^{\varepsilon}(Q)$ is a proper closed subset of the affine space $\mathscr{P}_{n}^{\varepsilon}(Q)$. 
Next we apply these results to $\mathrm{w}_{n}$.

THEOREM 3.6. $Z \cap \mathrm{PP}_{n}^{\varepsilon}$ is nowhere dense in ${ }_{n}^{\mathrm{P}}{ }_{n}$.

This result is a consequence of two properties of affine space.

LEMMA 3.7. If $U$ is any nonempty open subset of $Q^{n}$, then $U$ contains a point of $\mathbf{Z}^{n}$.

Proof. We may assume $U=D(f), f \in Q\left[x_{1}, \ldots, x_{n}\right]$. A standard argument using the fact that $\mathbf{Z}$ is infinite, [Z-S, p. 38], shows that there exist $a_{1}, a_{2}, \ldots, a_{n} \in \mathbf{Z}$ with $f\left(a_{1}, a_{2}, \ldots, a_{n}\right) \neq 0$. Thus $\left(a_{1}, a_{2}, \ldots, a_{n}\right) \in U \cap \mathbf{Z}^{n}$.

LEMMA 3.8. Let $Z$ be a proper closed subset of $Q^{n}$. Let $f$ be a nontrivial polynomial in $Q\left[x_{1}, \ldots, x_{n}\right]$. Then $D f \cap \mathbf{Z}^{n} \cap\left(Q^{n}-Z\right) \neq \varnothing$.

Proof. $D f$ and $Q^{n}-Z$ are nonempty open subsets of $Q^{n}$. Irreducibility of $Q^{n}$ implies that $D f \cap\left(Q^{n}-Z\right) \neq \varnothing$. Lemma 3.7 shows that $D f \cap\left(Q^{n}-Z\right)$ contains a point with integral coordinates.

Proof of 3.6. Since $Z \cap \mathscr{P}_{n}^{\varepsilon}(Q)$ is a proper closed subset of $\mathscr{P}_{n}^{\varepsilon}(Q) \cong Q^{n-1}$, for any nontrivial polynomial $f$ in $Q\left[x_{1}, \ldots, x_{n-1}\right], D(f) \cap\left(\mathscr{P P}_{n}^{\varepsilon}(Q)-\mathscr{P}_{n}^{\varepsilon}(Q) \cap Z\right)$ contains an integral point by 3.8. That is, $D f \cap\left(\mathscr{P}_{n}^{\varepsilon}-\mathscr{P P}_{n}^{\varepsilon} \cap Z\right) \neq \varnothing$. Thus $Z \cap{ }_{{ }_{n}}{ }_{n}$ is nowhere dense in $\rho_{n} \varepsilon$. This complete the proof.

THEOREM 3.9. Let $S \subset \mathscr{P}_{n}$ denote the set $\{$ char $\phi\}$ where $\phi$ ranges over geometric automorphisms of a free group of rank $n$. Then $S$ is nowhere dense in $\mathscr{P}_{n}$ for $n \geqslant 3$.

Proof. By Stallings' theorem quoted in the introduction, if $p \in S$, then some pair of roots $(\lambda, \mu)$ of $p$ satisfy $(\lambda \mu)^{r}=1$ for some integer $r \geqslant 1$. Corollary 2.5 shows that $p \in Z$. Thus $S \subset Z \cap \mathscr{P}_{n}$. By Theorem $3.6 Z \cap \mathscr{P}_{n}$ is nowhere dense in $\stackrel{P}{n}_{n}$ for $n \geqslant 3$. It follows that $S$ is nowhere dense. This completes the proof.

4. Remarks. In [St] it is conjectured that the fixed point set of a $P V$ automorphism $\phi$ of a free group $F$ is trivial, where $\phi$ is called $P V$ if char $\phi$ has a root $\theta$ which is a $P V$ number of degree equal to rank $F$. Here is a counterexample to that conjecture as stated. Let $\phi: F(x, y) \rightarrow F(x, y)$ be given by $\phi(x)=x^{2} y, \phi(y)=x y$. Then char $\phi$ $=x^{2}-3 x+1$ with roots $(3 \pm \sqrt{5}) / 2$. Hence $\phi$ is $P V$. However $\phi\left(\left[x, y^{-1}\right]\right)=$ $\left[x, y^{-1}\right]$, where $[a, b]=: a b a^{-1} b^{-1}$.

Of course one could simply patch up Stallings' conjecture by demanding that $F$ be of rank $\geqslant 3$. However there is a more elegant way of proceeding in light of our results.

We define an automorphism $\phi$ of a finitely generated free group $F$ to be generic if, for any pair of eigenvalues $(\lambda, \mu)$ of char $\phi$ and integer $r \geqslant 1,(\lambda \mu)^{r} \neq 1$. By our Theorem 2.4, the generic automorphisms are defined by an open condition in the Zariski topology. We observe also that if $\phi$ is generic, so is $\phi^{r}, r \neq 0$, and $\psi \phi \psi^{-1}$, where $\psi \in \operatorname{Aut}(F)$. Thus if $\phi$ is generic, so is the entire conjugacy class of $\phi$. Observe also that a $P V$ automorphism of $F$ of rank at least three is generic whereas no automorphism of a free group of rank two is generic. 
We should like to inquire then whether a generic automorphism of a free group has only trivial fixed points. There is some evidence for this $[\mathbf{S q}, \mathbf{G}]$. Examples are very hard to come by and difficult to compute.

\section{REFERENCES}

[St] J. R. Stallings, Topologically unrealizable automorphisms of free groups, Proc. Amer. Math. Soc. 84 (1982), 21-24.

[W] B. L. van der Waerden, Modern algebra, Vol. I, Ungar, New York, 1950.

[D] J. Dieudonné, The work of Bourbaki during the last thirty years, Notices Amer. Math. Soc. 29 (1982).

[Z-S] O. Zariski and P. Samuel, Commutative algebra, Vol. I, Van Nostrand, New York, 1958.

[L-S] R. C. Lyndon and P. E. Schupp, Combinatorial group theory, Ergebnisse der Math. Grenzgebiete, No. 89, Springer-Verlag, New York and Berlin, 1977.

[G] S. M. Gersten, On fixed points of certain automorphisms of free groups, Proc. London Math. Soc. (to appear)

[Sq] C. Squier, Fixed points and finite orbits of free group automorphisms, preprint.

[Si] C. L. Siegel, Algebraic integers whose conjugates lie in the unit circle. Duke Math. J. 11 (1944), 597-602.

Department of Mathematics, University of Utah, Salt lake City, Utah 84112 